\title{
VSI electromagnetic fields
}

\author{
Marcello Ortaggio and Vojtěch Pravda \\ Institute of Mathematics of the Czech Academy of Sciences \\ Žitná 25, 11567 Prague 1, Czech Republic \\ *E-mail: ortaggio(at)math(dot)cas(dot)cz, pravda@math.cas.cz
}

\begin{abstract}
A $p$-form $\boldsymbol{F}$ is VSI (i.e., all its scalar invariants of arbitrary order vanish) in a $n$ dimensional spacetime if and only if it is of type $\mathrm{N}$, its multiple null direction $\ell$ is "degenerate Kundt", and $£_{\ell} \boldsymbol{F}=0$. This recent result is reviewed in the present contribution and its main consequences are summarized. In particular, a subset of VSI Maxwell fields possesses a universal property, i.e., they also solve (virtually) any generalized (non-linear and with higher derivatives) electrodynamics, possibly also coupled to Einstein's gravity.
\end{abstract}

\section{Introduction}

The present contribution is summary of the main results of our recent work ${ }^{1}$. It is useful to start by defining the VSI property for a general tensor, i.e.,

Definition 1.1 (VSI tensors). A tensor in an $n$-dimensional spacetime with metric $g_{a b}$ is VSI $I_{I}$ if all the scalar polynomial invariants constructed from the tensor itself and its covariant derivatives up to order $I(I=0,1,2,3, \ldots)$ vanish. It is VSI if all its scalar polynomial invariants of arbitrary order vanish.

As a generalization of the notion of null fields $\underline{\underline{2}}$ to arbitrary $p$-forms, it is natural to introduce the following

Definition 1.2 ( $p$-forms of type $\mathbf{N}$ ). At a spacetime point, a p-form $\boldsymbol{F}$ is of type $N$ if it satisfies

$$
\ell^{a} F_{a b_{1} \ldots b_{p-1}}=0, \quad \ell_{[a} F_{\left.b_{1} \ldots b_{p}\right]}=0,
$$

where $\boldsymbol{\ell}$ is a null vector (this follows from (11) and need not be assumed). The second condition can be equivalently replaced by $\ell^{a *} F_{a b_{1} \ldots b_{n-p-1}}=0$.

This is equivalent to the type $\mathrm{N}$ condition in the set-up of $\mathrm{f}^{3}$.

First, with the results of $\frac{4}{\underline{4}}$, it is easy to see that a p-form $\boldsymbol{F}$ is VSI $I_{0}$ iff it is is of type $N$. Next, our main result is the VSI condition, given in the next section.

\section{Main result: VSI $p$-forms}

The main result of $\frac{1}{1}$ is the following

Theorem 2.1 (VSI $p$-forms $\mathbf{1}$ ). The following two conditions are equivalent:

(1) a non-zero $p$-form field $\boldsymbol{F}$ is VSI in a spacetime with metric $g_{a b}$

(2) (a) $\boldsymbol{F}$ possesses a multiple null direction $\boldsymbol{\ell}$, i.e., it is of type $N$

(b) $£_{\ell} \boldsymbol{F}=0$ 
(c) $g_{a b}$ is a degenerate Kundt metric, and $\boldsymbol{\ell}$ is the corresponding Kundt null direction.

We observe that, in Theorem 2.1, the $p$-form $\boldsymbol{F}$ is not assumed to satisfy any particular field equations and the result is thus purely geometric (on the other hand, if $\boldsymbol{F}$ is taken to be closed, i.e., $\mathrm{d} \boldsymbol{F}=0$, then condition $2 \mathrm{~b}$ automatically follows from the type $\mathrm{N}$ condition $2 \mathrm{a}$, and need not be assumed).

\subsection{Adapted coordinates}

From Theorem 2.1 with the results of $\frac{5,6}{2}$, it follows that coordinates $\left(u, r, x^{\alpha}\right)$, adapted to $\ell=\partial_{r}$, exist such that any VSI $p$-form can be written as

$$
\boldsymbol{F}=\frac{1}{(p-1) !} f_{\alpha_{1} \ldots \alpha_{p-1}}(u, x) \mathrm{d} u \wedge \mathrm{d} x^{\alpha_{1}} \wedge \ldots \wedge \mathrm{d} x^{\alpha_{p-1}}
$$

and the corresponding background metric as

$$
\begin{aligned}
& \mathrm{d} s^{2}=2 \mathrm{~d} u\left[\mathrm{~d} r+H(u, r, x) \mathrm{d} u+W_{\alpha}(u, r, x) \mathrm{d} x^{\alpha}\right]+g_{\alpha \beta}(u, x) \mathrm{d} x^{\alpha} \mathrm{d} x^{\beta}, \\
& W_{\alpha}(u, r, x)=r W_{\alpha}^{(1)}(u, x)+W_{\alpha}^{(0)}(u, x), \\
& H(u, r, x)=r^{2} H^{(2)}(u, x)+r H^{(1)}(u, x)+H^{(0)}(u, x),
\end{aligned}
$$

where $\alpha, \beta=2 \ldots n-1$, and $x$ denotes collectively the set of coordinates $x^{\alpha}$.

The degenerate Kundt metric (3)-(15) includes, e.g., all VSI spacetimes ${ }^{7.8}$, all $p p$ waves, and all Kundt Einstein (or aligned pure radiation) spacetimes - in particular, spacetimes of constant curvature (Minkowski and (A)dS). The corresponding Weyl and Ricci tensors are both of aligned type II, in general.

If $\boldsymbol{F}$ in (2) is required to obey the source-free Maxwell equations $\mathrm{d} \boldsymbol{F}=0=\mathrm{d}^{*} \boldsymbol{F}$, the following conditions are obtained

$$
f_{\left[\alpha_{2} \ldots \alpha_{p-1}, \alpha_{1}\right]}=0, \quad\left(\sqrt{\tilde{g}} f^{\beta \alpha_{1} \ldots \alpha_{p-2}}\right)_{, \beta}=0,
$$

where $\tilde{g} \equiv \operatorname{det} g_{\alpha \beta}=-\operatorname{det} g_{a b} \equiv-g$. These are Maxwell's equations for the $(p-1)$ form $\boldsymbol{f}$ in the $(n-2)$-dimensional Riemannian geometry associated with $g_{\alpha \beta}$, i.e., $\boldsymbol{f}$ must be harmonic (recall, however, that $\boldsymbol{f}$ can also depend on $u$ ). For $n=3,4$, it can be argued that to any $\boldsymbol{F}$ which is VSI, it can always be associated a solution of the Maxwell equations which is also VSI ${ }^{1}$. It is also interesting to observe that the effective Maxwell equations (6) are "immune" to adding a Chern-Simons term (except when this is linear, see $\underline{1}$ for details) to the full Maxwell equations.

\subsection{Universal solutions of generalized electrodynamics}

It was already known to Schrödinger $\frac{9,10}{9}$ that all null Maxwell fields (with $n=4=$ $2 p$ ) solve the equations for the electromagnetic field in any non-linear electrodynamics. More generally, we observe that a subset of the VSI Maxwell fields described above possesses a "universal" property, i.e., they solve simultaneously any electrodynamics whose field equations can be expressed as $\mathrm{d} \boldsymbol{F}=0, * \mathrm{~d} * \tilde{\boldsymbol{F}}=0$, where $\tilde{\boldsymbol{F}}$ 
can be any $p$-form constructed from $\boldsymbol{F}$ and its covariant derivatives. For example, any VSI Maxwell $\boldsymbol{F}$ is universal if the background is a Kundt spacetime of Weyl and traceless-Ricci type III (aligned) with $D R=0=\delta_{i} R$ (an affine parameter and a frame parallelly transported along $\ell$ are assumed - cf. 1 for the notation employed here). In particular, Ricci flat and Einstein Kundt spacetimes of Weyl type III/N/O can occur, the latter including Minkowski and (A)dS.

An explicit simple example is given by the Maxwell field

$$
\boldsymbol{F}=e^{x / 2} c(u) \mathrm{d} u \wedge\left(-\cos \frac{y e^{u}}{2} \mathrm{~d} x+e^{u} \sin \frac{y e^{u}}{2} \mathrm{~d} y\right),
$$

defined in the type III vacuum spacetime found by Petrov (eq. (31.40) in ${ }^{11}$ )

$$
\mathrm{d} s^{2}=2 \mathrm{~d} u\left[\mathrm{~d} r+\frac{1}{2}\left(x r-x e^{x}\right) \mathrm{d} u\right]+e^{x}\left(\mathrm{~d} x^{2}+e^{2 u} \mathrm{~d} y^{2}\right) .
$$

More general results will be presented elsewhere.

\section{Einstein-Maxwell solutions}

\subsection{General field equations}

The previous discussion applies to VSI test fields, since we have not considered the consequences of the backreaction on the spacetime geometry. In the full EinsteinMaxwell theory with an arbitrary cosmological constant $\Lambda$, one finds that the metric functions entering (3) must satisfy the following set of equations (obtained in 1 refining the results of ${ }^{12}$ )

$$
\begin{aligned}
& \mathcal{R}_{\alpha \beta}=\frac{2 \Lambda}{n-2} g_{\alpha \beta}+\frac{1}{2} W_{\alpha}^{(1)} W_{\beta}^{(1)}-W_{(\alpha \| \beta)}^{(1)}, \\
& 2 H^{(2)}=\frac{\mathcal{R}}{2}-\frac{n-4}{n-2} \Lambda+\frac{1}{4} W^{(1) \alpha} W_{\alpha}^{(1)}, \\
& W_{\alpha \| \beta}^{(1) \beta}=\frac{1}{2} W^{(1) \beta}\left(3 W_{\alpha \| \beta}^{(1)}-W_{\beta \| \alpha}^{(1)}\right)+W_{\alpha}^{(1)}\left(W_{\| \beta}^{(1) \beta}-\frac{1}{2} W^{(1) \beta} W_{\beta}^{(1)}-\frac{2 \Lambda}{n-2}\right),(11) \\
& 2 H_{, \alpha}^{(1)}=-g_{\alpha \beta, u}{ }^{\| \beta}+2 W_{[\alpha \| \beta]}^{(0)}{ }_{\beta}-2 W^{(0) \beta} W_{\alpha \| \beta}^{(1)}+\left(W^{(0) \beta} W_{\beta}^{(1)}\right)_{, \alpha}+W_{\alpha, u}^{(1)}+2(\ln \sqrt{\tilde{g}})_{, u \alpha} \\
& \quad+W_{\alpha}^{(1)}\left[W^{(0) \beta} W_{\beta}^{(1)}-W^{(0) \beta} \|_{\| \beta}+(\ln \sqrt{\tilde{g}})_{, u}\right]+\frac{4 \Lambda}{n-2} W_{\alpha}^{(0)}, \\
& \Delta H^{(0)}+W^{(1) \alpha} H_{, \alpha}^{(0)}+W^{(1) \alpha} \|_{\| \alpha} H^{(0)}=W^{(0) \beta} W_{\beta}^{(0)}\left(\frac{1}{2} W^{(1) \alpha}{ }_{\| \alpha}-\frac{2 \Lambda}{n-2}\right) \\
& \quad+H^{(1)}\left[W^{(0) \alpha}-(12)\right. \\
& \quad-W^{(0) \beta}\left(2 W^{(1) \alpha} W_{[\alpha \| \beta]}^{(0)}+W_{\beta, u}^{(1)}-2 H_{, \beta}^{(1)}\right)-(\ln \sqrt{\tilde{g}})_{, u u}+\frac{1}{4} g_{, u}^{\alpha \beta}{ }_{, u} g_{\alpha \beta, u}-W_{0}^{(0) \alpha} \mathcal{F}^{2} .
\end{aligned}
$$

Heree $\mathcal{R}_{\alpha \beta}, \mathcal{R}$ and $\|$ denote, respectively, the Ricci tensor, the Ricci scalar and the covariant derivative associated with $g_{\alpha \beta}, W^{(1) \alpha} \equiv g^{\alpha \beta} W_{\beta}^{(1)}, \Delta$ is the Laplace operator in the geometry of the transverse metric $g_{\alpha \beta}$, and $\kappa_{0}$ is a gravitational coupling constant. The Maxwell equations (6) must also be satisfied. 
The simplest examples one can construct are electromagnetic and gravitational "plane-fronted" waves (with $W_{\alpha}^{(0)}=0$ ) propagating in a constant curvature background, giving rise to Kundt waves of Weyl type $\mathrm{N}$ (in four dimensions see, e.g., 13,14 $)$. More general (e.g., with $W_{\alpha}^{(0)} \neq 0$ ) degenerate Kundt metrics with null Maxwell fields are also known (see 11,15,16 and references therein for $n=4$ ). The case of VSI $p$-form Maxwell fields in VSI and $p p$-waves spacetimes have been discussed in $\underline{1}$ (where further references can be found).

\subsection{Universal Einstein-Maxwell solutions}

Some of the universal Maxwell fields mentioned in section 2.2 can also be used to construct exact solutions of full general relativity, where the energy-momentum tensor $T_{a b}$ associated with the electromagnetic field is determined in the generalized electrodynamics (in terms of $\boldsymbol{F}$ and its covariant derivatives - cf. 17 for an example). For example, as pointed out in $\underline{1}$, all VSI spacetimes with $L_{i 1}=0=L_{1 i}$ (i.e., the recurrent ones) coupled to an aligned VSI $p$-form field that solve the standard Einstein-Maxwell equations are also exact solutions of gravity coupled to generalized electrodynamics, provided $p>1$ and $\delta_{i} F_{1 j_{1} \ldots j_{p-1}}=0$ (in an "adapted" parallely transported frame, i.e., such that $\stackrel{i}{j}_{j k}=0$ ). Within this family, metrics of Weyl type $\mathrm{N}$ are necessarily $p p$-waves, for which such a universal property was pointed out in $\underline{18}-\underline{20}$, at least for certain values of $p$. But metrics of Weyl type III are also permitted, including $p p$-waves $\left(L_{11}=0\right)$ and also genuinely recurrent $\left(L_{11} \neq 0\right)$ spacetimes (for $n=4, p=3$ this was discussed in ${ }^{21}$ ). One explicit example of the latter solutions in $4 \mathrm{D}$ is given by the Maxwell field (17) with the metric

$$
\mathrm{d} s^{2}=2 \mathrm{~d} u\left[\mathrm{~d} r+\frac{1}{2}\left(x r-x e^{x}-2 \kappa_{0} e^{x} c^{2}(u)\right) \mathrm{d} u\right]+e^{x}\left(\mathrm{~d} x^{2}+e^{2 u} \mathrm{~d} y^{2}\right),
$$

which is a modification of (8) taking into account the backreaction.

As in section 2.2, the above discussion applies to generalized electrodynamics with arbitrary higher-order derivative "corrections". A special instance of this result is the fact that Einstein-Maxwell solutions with aligned null electromagnetic fields (not necessarily VSI) are also solution of NLE coupled to gravity, as previously demonstrated in ${ }^{22}-24$.

\section{Further remarks}

For certain purposes, it may be useful to observe that if $\boldsymbol{F}$ is $\mathrm{VSI}_{3}$ then it is necessarily VSI (this follows from the proof of Theorem 2.1 given in $\underline{\underline{1}}$ ). For completeness, let us thus also give the necessary and sufficient conditions for a $p$-form $\boldsymbol{F}$ to be $\mathrm{VSI}_{1}$ or $\mathrm{VSI}_{2}$ (recall that $\mathrm{VSI}_{0}$ means type $\mathrm{N}$, as mentioned in section 11):

Proposition 4.1 ( $\mathbf{V S I}_{1}$ and $\mathbf{V S I}_{2} p$-forms ${ }^{\mathbf{1}}$ ). A $p$-form $\boldsymbol{F}$ is $V S I_{1}$ iff it is is of type $N, £_{\boldsymbol{\ell}} \boldsymbol{F}=0, \boldsymbol{\ell}$ is Kundt. It is $V_{S I_{2}}$ iff it is is of type $N, £_{\boldsymbol{\ell}} \boldsymbol{F}=0, \boldsymbol{\ell}$ is Kundt and (at least) doubly aligned with the Riemann tensor. 


\begin{tabular}{|c|l|l|l|}
\hline & vector $\boldsymbol{\ell}$ & \multicolumn{1}{|c|}{$p$-form $\boldsymbol{F}$} & Riemann \\
\hline $\mathrm{VSI}_{0}$ & null & N & III (N,O) \\
\hline $\mathrm{VSI}_{1}$ & Kundt & N, $£_{\boldsymbol{\ell}} \boldsymbol{F}=0$, Kundt & $(*)$ N, $\kappa=0, \sigma \Psi_{4}=\rho \Phi_{22}$ \\
\hline $\mathrm{VSI}_{2}$ & Kundt, Riem II & N, $£_{\boldsymbol{\ell}} \boldsymbol{F}=0$, Kundt, Riem II & III, Kundt \\
\hline $\mathrm{VSI}_{3}$ & degKundt & N, $£_{\boldsymbol{\ell}} \boldsymbol{F}=0$, degKundt & “ \\
\hline$\vdots$ & " & " & “ \\
\hline $\mathrm{VSI}$ & " & " & " \\
\hline
\end{tabular}

Fig. 1. Comparing various $\mathrm{VSI}_{I}$ conditions for a 1 -form $\boldsymbol{\ell}$, a $p$-form $\boldsymbol{F} \underline{1}$ and the Riemann tensor $7,8,25$. The quotation marks " mean that the same conditions as in the next-upper row apply. The Riemann $\mathrm{VSI}_{1}$ condition $(*)$ needs some comments for two reasons. First, it has been investigated only in the case $n=4^{25}$ (and indeed it is given above in the standard 4D NP notation). Additionally, it is sufficient but not necessary (contrary to the rest of the table): also Kundt spacetimes of Riemann type III are $\mathrm{VSI}_{1}$ (but not "properly", i.e., they are in fact VSI, cf. the lower rows in the same column).

It is also interesting to observe that Theorem 2.1 and Proposition 4.1 apply also in the limiting case $p=1$, i.e., when $\boldsymbol{F}$ is a vector field. In particular, when $p=1$, Theorem 2.1 reduces to: a vector field $\boldsymbol{\ell}$ is VSI in a spacetime with metric $g_{a b}$ iff $\boldsymbol{\ell}$ is Kundt and affinely parameterized, and $g_{a b}$ is a degenerate Kundt metric w.r.t $\boldsymbol{\ell}$.

The above comments are summarized in the first two columns of Fig. 1 The last column gives corresponding results in the case of the Riemann tensors (i.e., for the $\mathrm{VSI}_{I}$ spacetimes) $)^{7,8,25}$. While certain conditions turn out to be similar, there is also an important difference: for the Riemann tensor one has already $V S I_{2} \Rightarrow V S I$.

\section{Acknowledgments}

We thank research plan RVO: 67985840 and research grant GAČR 13-10042S.

\section{References}

1. M. Ortaggio and V. Pravda, Electromagnetic fields with vanishing scalar invariants, Class. Quantum Grav. 33, p. 115010 (2016).

2. J. L. Synge, Relativity: the Special Theory (North-Holland, Amsterdam, 1955).

3. R. Milson, A. Coley, V. Pravda and A. Pravdová, Alignment and algebraically special tensors in Lorentzian geometry, Int. J. Geom. Meth. Mod. Phys. 2, 41 (2005).

4. S. Hervik, A spacetime not characterized by its invariants is of aligned type II, Class. Quantum Grav. 28, p. 215009 (2011).

5. A. Coley, S. Hervik and N. Pelavas, Spacetimes characterized by their scalar curvature invariants, Class. Quantum Grav. 26, p. 025013 (2009). 
6. A. Coley, S. Hervik, G. O. Papadopoulos and N. Pelavas, Kundt spacetimes, Class. Quantum Grav. 26, p. 105016 (2009).

7. V. Pravda, A. Pravdová, A. Coley and R. Milson, All spacetimes with vanishing curvature invariants, Class. Quantum Grav. 19, 6213 (2002).

8. A. Coley, R. Milson, V. Pravda and A. Pravdová, Vanishing scalar invariant spacetimes in higher dimensions, Class. Quantum Grav. 21, 5519 (2004).

9. E. Schrödinger, Contributions to Born's new theory of the electromagnetic field, Proc. Roy. Soc. London Ser. A 150, 465 (1935).

10. E. Schrödinger, A new exact solution in non-linear optics (two-wave-system), Proc. Roy. Irish Acad. A49, 59 (1943).

11. H. Stephani, D. Kramer, M. MacCallum, C. Hoenselaers and E. Herlt, Exact Solutions of Einstein's Field Equations, second edn. (Cambridge University Press, Cambridge, 2003).

12. J. Podolský and M. Žofka, General Kundt spacetimes in higher dimensions, Class. Quantum Grav. 26, p. 105008 (2009).

13. A. García Díaz and J. F. Plebański, All nontwisting N's with cosmological constant, J. Math. Phys. 22, 2655 (1981).

14. I. Ozsváth, I. Robinson and K. Rózga, Plane-fronted gravitational and electromagnetic waves in spaces with cosmological constant, J. Math. Phys. 26, 1755 (1985).

15. J. B. Griffiths and J. Podolský, Exact Space-Times in Einstein's General Relativity (Cambridge University Press, Cambridge, 2009).

16. J. Griffiths, P. Docherty and J. Podolský, Generalized Kundt waves and their physical interpretation, Class. Quantum Grav. 21, 207 (2004).

17. B. Podolsky, A generalized electrodynamics. Part I- Non-quantum, Phys. Rev. 62, 68 (1942).

18. R. Güven, Plane waves in effective theories of superstrings, Phys. Lett. B 191, 275 (1987).

19. G. T. Horowitz and A. R. Steif, Spacetime singularities in string theory, Phys. Rev. Lett. 64, 260 (1990).

20. G. T. Horowitz, Singularities in string theory, in Strings '90, eds. R. Arnowitt, R. Bryan, M. J. Duff, D. Nanopoulos, C. N. Pope and E. Sezgin (World Scientific, Singapore, 1990) pp. 163-175.

21. A. A. Coley, A class of exact classical solutions to string theory, Phys. Rev. Lett. 89, p. 281601 (2002).

22. S. Kichenassamy, Sur le champ électromagnétique singulier en théorie de BornInfeld, C. R. Hebd. Seanc. Acad. Sci. 248, 3690 (1959).

23. H. Kremer and S. Kichenassamy, Sur le champ électromagnétique singulier dans une théorie du type Born-Infeld, C. R. Hebd. Seanc. Acad. Sci. 250, 1192 (1960).

24. A. Peres, Nonlinear electrodynamics in general relativity, Phys. Rev. 122, 273 (1961). 
25. N. Pelavas, A. Coley, R. Milson, V. Pravda and A. Pravdová, VSI $i_{i}$ spacetimes and the $\epsilon$-property, J. Math. Phys. 46, p. 063501 (2005). 